\title{
A Tensor Model of Multipath Routing Based on Multiple QoS Metrics
}

\author{
Olexandr V. Lemeshko, Oksana Yu. Yevseyeva O., Sergey V. Garkusha
}

\begin{abstract}
Flow model for multipath routing with guaranteed the quality of service $(\mathrm{QoS})$ is proposed. The model is based on the updated version of flow conservation law, which is taking into account the possible packet loss caused by buffer overflow at the network routers. The accounting such losses is related to revise conditions associated with the quality of service. By using tensor approach analytical boundary conditions were obtained. The conditions allow ensuring different parameters of the quality of service (rate, average delay and packet loss probability) at the same time. In addition, proposed model provides minimal value of average end-to-end packet delay where delays along all paths are same. As a result jitter caused by the multipath routing (difference between delays along different paths) is minimized. The developed conditions for QoS ensuring have invariant form which do not depend on the used at the interface. Changing the traffic models and/or packet servicing algorithm is related to a modification of the metric only within the same form of conditions.
\end{abstract}

Index Terms-tensor model, routing, quality of service, packet loss.

\section{INTRODUCTION}

$\mathrm{T}$ he development of modern multiservice telecommunications systems (TCS) is related to improvement of different tools for QoS ensuring. The achieved QoS level is a major criterion for the competitive selection of a telecommunications technology for existing and projected TCS. It is known, that modern multimedia traffic has set of requirements (of average delay, jitter, packet loss and packet transmission rate) which must be satisfied simultaneously. In addition, depending on the type (class) of servicing requirements to the numerical values of QoS-metrics may differ materially [1]. It is leading to complication of QoS ensuring problem.

Though all of the levels of the OSI model cause the result quality, from viewpoint of end-to-end QoS key role belongs to tools of control traffic at the network level of OSI, where routing takes a main stand [1]. As a result we have actual problem of QoS-routing, which consists in finding an optimal cost path from a source to

A. V. Lemeshko is with the Kharkov National University of Radioelektronics, Kharkov, 61166, Ukraine (e-mail: avlem@mail.ru).

O. Yu. Yevseyeva is with the Kharkov National University of Radioelektronics, Kharkov, 61166, Ukraine (e-mail: evseeva.o.yu@gmail.com).

S. V. Garkusha is with the Poltava University Economics and Trade, Poltava, 36014, Ukraine (e-mail: sv.garkusha@mail.ru). a destination subject to one or more QoS-constraints on the path. In order to improve efficiency of TCS we'll focus on multipath routing [1].

On another hand mathematical model on multipath QoS-routing must take into account flow nature of real telecommunications traffic. As a result absence of effective models of multipath QoS-routing causes developing of new models which (1) are able to take into account set of QoS-metrics simultaneously, (2) are flowbased, (3) allow finding optimal solution of the problem. In this paper we provide such model for the QoS-routing which was developed within tensor approach. Within the model necessary and sufficient conditions for QoS ensuring were obtained.

\section{A FLOW-BASED ROUTING MODEL WITH PACKET LOSS}

Within the routing model structure of TCS will be described by a one-dimensional network $S=(U, V)$, where $U=\left\{u_{i}, i=\overline{1, m}\right\}$ is set of nodes (routers) in the network, $V=\left\{v_{z}=(i, j) ; z=\overline{1, n} ; i, j=\overline{1, m} ; i \neq j\right\}$ is set of edges (arcs). Here the edge $v_{z}=(i, j) \in V$ models $z$-th link connected router $i$ to router $j$. Assume capacity $\varphi_{(i, j)}$ of every link $(i, j)$ measured as number of packets per second is known.

Each router has multiple interfaces, through which it transmits packets to adjacent nodes (the neighbors). Assume the number of interface corresponds to the number of neighboring node which has been connected through the interface. The result of the routing problem solving is the calculation of the set of routing variables $x_{(i, j)}^{k}$, each of which characterizes the fraction of the intensity of the $k$-th traffic directed from the node $i$ to the node $j$ via an appropriate, i.e. $j$-th interface (fig. $1)$.

In process of the TCS functioning queue overflow raises packet losses on the interfaces of nodes (routers). Let us use $p_{(i, j)}^{k}$ to note the probability of packet loss on the $(i, j)$ interface where the lost packets were part of $k$-th traffic. Then expression $x_{(i, j)}^{k}\left(1-p_{(i, j)}^{k}\right)$ defines fraction of the intensity of the $k$-th traffic transmitted through link $(i, j)$. But $x_{(i, j)}^{k} p_{(i, j)}^{k}$ is fraction of the $k$-th traffic dropped on $j$-th interface of node $i$ (fig. 1). 


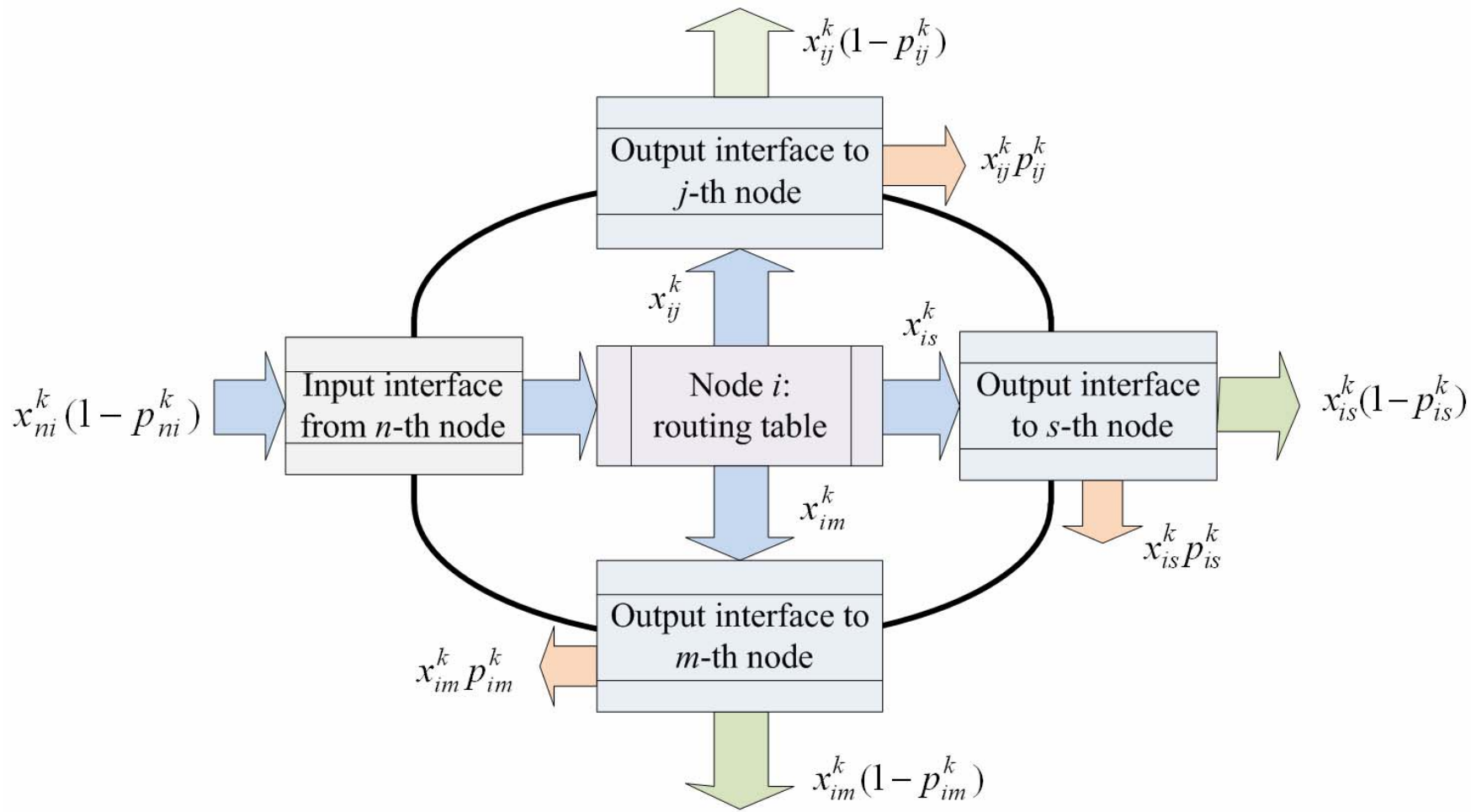

Fig. 1 The simplified architecture of the network node from viewpoint of the routing of $k$-th traffic

In order to take into account the lost packets on nodes of TCS law of flow conservation must be rewritten in new form:

$$
\left\{\begin{array}{l}
\sum_{j:(i, j) \in V} x_{(i, j)}^{k}=1, k \in K, i=s_{k} ; \\
\sum_{j:(i, j) \in V} x_{(i, j)}^{k}-\sum_{j:(j, i) \in V} x_{(j, i)}^{k}\left(1-p_{(j, i)}^{k}\right)=0, k \in \mathrm{K}, i \neq s_{k}, d_{k} ; \\
\sum_{j:(j, i) \in V} x_{(j, i)}^{k}\left(1-p_{(i, j)}^{k}\right)=\varepsilon^{k}, k \in K, i=d_{k},
\end{array}\right.
$$

where $K$ is set of traffics in the network; $s_{k}$ is node-sender and $d_{k}$ is node-receiver for the $k$-th traffic; $\varepsilon^{k}$ is fraction of the $k$-th traffic serviced by the network, i.e. this fraction of transmitted packets delivered from the sending node to the receiving node successfully.

The solving of routing problem requires analytical representation of the interfaces. For the formal description of the interface we will use the results of queuing theory as one possible way to do it. For instance by using queuing system $M / M / 1 / N$ probability of packet loss can be calculated as

$$
p=p\left(q<\Theta_{b}\right)=\frac{(1-\rho)(\rho)^{N}}{1-(\rho)^{N+1}},
$$

where $\rho=\frac{\lambda}{\varphi}$ is utilization of the link; $q$ is current value of average length of queue; $N=\Theta_{b}+1$ is maximal number of packets, which can be on the interface, including buffer $\left(\Theta_{b}\right)$ and, actually, link; $\lambda$ is packet intensity of the total traffic transmitted through the link, $1 / \mathrm{s}$.
If take into account all possible packet losses the intensity of the traffic transmitted through the link $(i, j) \in V$ can be calculated as

$$
\lambda_{(i, j)}=\sum_{k \in K} \lambda_{k}^{\langle r e q\rangle} x_{(i, j)}^{k}\left(1-p_{(i, j)}^{k}\right),
$$

where $\lambda_{k}^{\langle r e q\rangle}$ is average intensity of the $k$-th traffic that is arriving into network. This value is required packet transmission rate, one of QoS-metrics.

In order to implement multipath routing strategy with load balancing the control (routing) variables $x_{(i, j)}^{k}$ must satisfy the condition

$$
0 \leq x_{(i, j)}^{k} \leq 1
$$

The precondition for controllability of routing is capacity constraint, i.e. the condition $\rho<1$, therefore the model must contain the following expression:

$$
\sum_{k \in K} \lambda_{k}^{\langle r e q\rangle} x_{(i, j)}^{k}<\varphi_{(i, j)}, \quad(i, j) \in E .
$$

Because of the random nature of the network traffic performance of constraints (5) is only a necessary, but not sufficient condition for the absence of packet loss on the nodes of TCS caused by possible buffer overflow.

\section{TENSOR MODEL OF MULTIPATH QOS-ROUTING BASED ON MULTIPLE METRICS}

In order to obtain a tensor model of the TCS let us introduce anisotropic space-structure constructed by the set of 
closed and open paths (circuits and node pairs). The dimension of this space is determined by the total number of edges in the network, and is equal to $n$ [2]. Each independent path defines coordinate axis in the space-structure.

Assume $S$ is a connected network, i.e. contains one connected component, then the cyclomatic number $\mu(S)$ and rank $\phi(S)$ of the network define the number of basic circuits and node pairs, determining the validity of the following expressions:

$$
\phi(S)=m-1, \mu(S)=n-m+1, n=\phi(S)+\mu(S) .
$$

From all possible circuits let us choose $\mu(S)$ of linearly independent circuits $\left\{\pi_{i}, i=\overline{1, \mu}\right\}$; set of all node pairs we will note as $\left\{\eta_{j}, j=\overline{1, \phi}\right\}$. These sets form basis for $n$-dimension space, corresponding to the structure of the network.

In introduced $n$-dimension space, the TCS can be described by mixed divalent tensor

$$
Q=T \otimes \Lambda,
$$

where $\otimes$ is sign of direct tensor multiplication, and components of tensor $Q$ are univalent covariant tensor of average packet delay $T$ and univalent contravariant tensor of traffic intensity $\Lambda$.

Then, the expression (7) can be written in index form

$$
q_{j}^{i}=\tau_{j} \lambda^{i}, i, j=\overline{1, n},
$$

where $\tau_{j}$ is packet transmission delay along the $j$-th coordinate path $(\mathrm{s}) ; \lambda^{i}$ is packet intensity of traffic transmitted along $i$-th coordinate path $(1 / \mathrm{s})$.

To consider the tensor (7) we will take into account the following two coordinate systems (CS): coordinate system of the edges (type $v$ ) and the coordinate system of the circuits and node pairs (type $\pi \eta$ ). Within tensor approach one of key role belongs to the variance of used tensors. The variance of a tensor is related to rules of transformation of its coordinates when transition from one CS to another. The variance of components of the tensor (8) is proved in [3]. It is known that the characteristics (metrics) which are submitting to conservation law, for example, the intensity of the traffic, are contravariant values, and the additive parameters, for example, the average packet delay, are covariant values.

In the framework of the chosen example, when the interface is modeled as a queuing system with losses $M / M / 1 / N$, the average packet delay in any communication link is approximated by

$$
\tau=\frac{\rho-\rho^{N+2}-(N+1) \rho^{N+1}(1-\rho)}{\lambda\left(1-\rho^{N+1}\right)(1-\rho)} .
$$

For further tensor generalization the expression (9) can be written in the form

$$
\tau_{i}^{v}=\frac{\rho_{i}^{v}-\left(\rho_{i}^{v}\right)^{N_{i}^{v}+2}-\left(N_{i}^{v}+1\right)\left(\rho_{i}^{v}\right)^{N_{i}^{v}+1}\left(1-\rho_{i}^{v}\right)}{\left(1-\left(\rho_{i}^{v}\right)^{N_{i}^{v}+1}\right)\left(1-\rho_{i}^{v}\right)\left(\lambda_{i}^{v}\right)^{2}} \lambda_{v}^{i},
$$

where $i=\overline{1, n}$ shows number of link in TCS, but index $\mathrm{V}$ shows type of coordinate system (all parameters in (10) belong to edges where every edge models some link).
In accordance with the Kron's postulate of the second generalizations [2], a system of equations (10) can be replaced by the following vector equation

$$
\Lambda_{v}=G_{v} T_{v},
$$

where $\Lambda_{v}$ and $T_{v}$ are projections of tensor $\Lambda$ and tensor $T$ in CS of edges respectively, $\Lambda_{v}$ and $T_{v}$ are $n$-dimensional vectors of traffic intensity and average packet delay in edges of the network respectively; $G_{v}=\left\|g_{v}^{i j}\right\|$ is $n \times n$ diagonal matrix, diagonal elements of which are calculated according to (10), referred to the appropriate edges $\left\{v_{i}, i=\overline{1, n}\right\}$, i.e.

$$
g_{v}^{i j}=\frac{\left(1-\left(\rho_{i}^{v}\right)^{N_{i}^{v}+1}\right)\left(1-\rho_{i}^{v}\right)\left(\lambda_{i}^{v}\right)^{2}}{\rho_{i}^{v}-\left(\rho_{i}^{v}\right)^{N_{i}^{v}+2}-\left(N_{i}^{v}+1\right)\left(\rho_{i}^{v}\right)^{N_{i}^{v}+1}\left(1-\rho_{i}^{v}\right)} .
$$

According to definition [2] the coordinate transformation rules for tensors are linear and can be formalized by a nonsingular square $n \times n$ matrix $C$ :

$$
\Lambda_{v}=C \Lambda_{\pi \eta},
$$

where $\Lambda_{\pi \eta}$ is $n$-dimensional vectors that are projection of tensor $\Lambda$ in coordinate system of circuits and node pairs (type $\pi \eta)$. In turn, the vector $\Lambda_{\pi \eta}$ has components

$$
\Lambda_{\pi \eta}=\left[\begin{array}{c}
\Lambda_{\pi} \\
-- \\
\Lambda_{\eta}
\end{array}\right] ; \Lambda_{\pi}=\left[\begin{array}{l}
\lambda_{\pi}^{1} \\
\vdots \\
\lambda_{\pi}^{j} \\
\vdots \\
\lambda_{\pi}^{\mu}
\end{array}\right] ; \Lambda_{\eta}=\left[\begin{array}{c}
\lambda_{\eta}^{1} \\
\vdots \\
\lambda_{\eta}^{p} \\
\vdots \\
\lambda_{\eta}^{\phi}
\end{array}\right],
$$

where $\Lambda_{\pi}$ is $\mu$-dimensional subvector related to traffic intensities into circuits of the network; $\Lambda_{\eta}$ is $\phi$-dimensional subvector related to traffic intensities between node pairs of the network; $\lambda_{\pi}^{j}$ is the traffic intensity in circuit $\pi_{j}$ of the network; $\lambda_{\eta}^{p}$ is the traffic intensity which is entering into the network and outgoing the network through the node pair $\eta_{p}$.

Projection of the tensor of average delays $T$ in a coordinate system of circuits and node pairs (type $\pi \eta$ ) is represented by the $\mathrm{n}$-dimensional vector with the following structure:

$$
T_{\pi \eta}=\left[\begin{array}{c}
T_{\pi} \\
-- \\
T_{\eta}
\end{array}\right] ; \quad T_{\pi}=\left[\begin{array}{c}
\tau_{1}^{\pi} \\
\vdots \\
\tau_{j}^{\pi} \\
\vdots \\
\tau_{\mu}^{\pi}
\end{array}\right] ; \quad T_{\eta}=\left[\begin{array}{c}
\tau_{1}^{\eta} \\
\vdots \\
\tau_{p}^{\eta} \\
\vdots \\
\tau_{\phi}^{\eta}
\end{array}\right],
$$

where $\tau_{j}^{\pi}, \tau_{p}^{\eta}$ are average packet delay in circuit $\pi_{j}$ and between node pair $\eta_{p}$ respectively. Sizes of $T_{\pi}$ and $T_{\eta}$ are $\mu$ and $\phi$ respectively.

Covariant character of delay tensor $T$ causes following law of the coordinate transformation:

$$
T_{v}=A T_{\pi \eta}
$$


where $A$ is $n \times n$ matrix of covariant coordinate transformation when transition from CS of circuits and node pairs to CS of edges.

The matrix $A$ is related to matrix $C$ (matrix of contravariant coordinate transformation) by orthogonality condition $C A^{t}=I ; \quad I \quad$ is unity $n \times n$ matrix, $[\cdot]^{t}$ is transposition sign.

Expression (11) has the same form in coordinate system of circuits and node pair:

$$
\Lambda_{\pi \eta}=G_{\pi \eta} T_{\pi \eta} .
$$

Then the tensor $G$ is divalent contravariant metric tensor:

$$
G_{\pi \eta}=A^{t} G_{v} A \text {, }
$$

where $G_{\pi \eta}$ is projection of tensor $G$ in CS of circuits and node pair.

Let us consider within the tensor model (6)-(18) the problem of conditions for QoS ensuring in TCS. Assume we have numerical requirements of average delay $\tau_{\langle\text {req }\rangle}$, packet loss probability $p_{\langle r e q\rangle}$ and packet transmission rate (intensity) $\lambda^{\langle r e q\rangle}$. Our goal is obtaining analytical conditions for ensuring all of the requirements. Let us agree that node pair source-destination will have the first number (14).

Under routing model represented by expressions (1)-(5) packet loss rate (intensity of lost packets) on node $\mathrm{u}_{\mathrm{i}}$ is calculated as

$$
\lambda_{\eta}^{i}=\sum_{j=1}^{R_{i}} \sum_{k \in K} \lambda_{k}^{\langle r e q\rangle} x_{(i, j)}^{k} p_{(i, j)}^{k},
$$

where $R_{i}$ is total number of output interfaces on routing node $i$.

Then the required conditions for QoS ensuring are

$$
\begin{gathered}
\sum_{j=2}^{\phi} \lambda_{\eta}^{j} \leq \lambda^{\langle r e q\rangle} p_{\langle r e q\rangle} . \\
\lambda^{\langle r e q\rangle}\left(1-p_{\langle r e q\rangle}\right) \leq G_{\pi \eta}^{\langle 4,2\rangle}\left[G_{\pi \eta}^{\langle 4,4\rangle}\right]^{-1} \Lambda_{\eta-1}+ \\
+\left(G_{\pi \eta}^{\langle 4,1\rangle}-G_{\pi \eta}^{\langle 4,2\rangle}\left[G_{\pi \eta}^{\langle 4,4\rangle}\right]^{-1} G_{\pi \eta}^{\langle 4,3\rangle}\right) \tau_{\langle r e q\rangle},
\end{gathered}
$$

where

$$
G_{\pi \eta}=\left\|\begin{array}{lll}
G_{\pi \eta}^{\langle 1\rangle} & \mid & G_{\pi \eta}^{\langle 2\rangle} \\
--- & + & --- \\
G_{\pi \eta}^{\langle 3\rangle} & \mid & G_{\pi \eta}^{\langle 4\rangle}
\end{array}\right\| ; G_{\pi \eta}^{\langle 4\rangle}=\left\|\begin{array}{lll}
G_{\pi \eta}^{\langle 4,1\rangle} & \mid & G_{\pi \eta}^{\langle 4,2\rangle} \\
--- & + & --- \\
G_{\pi \eta}^{\langle 4,3\rangle} & \mid & G_{\pi \eta}^{\langle 4,4\rangle}
\end{array}\right\| \text { and } G_{\pi \eta}^{\langle 1\rangle}
$$

are square $\phi \times \phi$ and $\mu \times \mu$ submatrices, respectively, $G_{\pi \eta}^{\langle 2\rangle}$ is $\mu \times \phi$ submatrix, $G_{\pi \eta}^{\langle 3\rangle}$ is $\phi \times \mu$ submatrix; $G_{\pi \eta}^{\langle 4,1\rangle}$ is the first element of the matrix $G_{\pi \eta}^{\langle 4\rangle}$.

Inequalities (19)-(20) are the desired conditions to ensure quality of service on set of heterogeneous metrics (transmission rate $\left(\lambda^{\langle r e q\rangle}\right)$, time $\left(\tau_{\langle\text {req }\rangle}\right)$ and reliability $\left.\left(p_{\langle\text {req }\rangle}\right)\right)$ simultaneously. In addition, the derivation of the inequalities (19)-(20) is based on requirement $T_{\pi}=0$. It means delay in every circuit must be zero. And it guarantees that delay in pair source-destination will be minimal and delays along different paths will be same. As a result jitter caused by multipath routing is minimized.

\section{CONCLUSION}

In this paper we offered the new tensor model for multipath routing with QoS guarantees. The model is based on renewed version of flow conservation law (1), which takes into account the possible packet losses caused by buffer overflow on the routers of TCS. The accounting of such losses requires reviewing conditions for QoS ensuring. Under the tensor model of TCS we obtained the analytical conditions for ensuring of transmission rate, average delay and packet loss probability simultaneously. In addition, the proposed solution provides the minimum average packet delay between source and destination where delays along different paths are same. It helps to minimize packet jitter caused by the multipath routing strategies.

It is important to note that the conditions to ensure the quality of service (19) and (20) have invariant form and don't depend on the used traffic and servicing models. Changing of the models affects modification of the metric (12) with preservation of the forms (19) and (20).

\section{REFERENCES}

[1] Vegesna S. IP Quality of Service / Srinivas Vegesna. Cisco Press. 2001. $368 \mathrm{p}$.

[2] Kron G. Tensor analysis of networks, Wiley, New York, 1939. 635 pp.

[3] Lemeshko A.V. Probabilistic-Temporal Model of QOS-Routing with Precomputation of Routes under the Terms of Non-Ideal Reliability of Telecommunication Network, Telecommunications and Radio Engineering, Vol. 66, Issue 13, 2007, pp. 1151-1166.

Olexandr V. Lemeshko received the Doctor's degree in telecommunication systems and networks from Kharkov National University of Radioelectronics, Ukraine, in 2005. From 2005 he is professor of department of Telecommunication Systems in Kharkov National University of Radioelectronics, and assistant editor of the journal "Problems of Telecommunications". His research interest includes the optimization and quality of service in telecommunications. He is the coauthor of three books, author of more than 100 articles, and more than 10 inventions.

Oksana Yu. Yevsyeyeva received the Doctor's degree in telecommunication systems and networks from Kharkov National University of Radioelectronics, Ukraine, in 2010. Currently she is professor of department of Telecommunication Systems in Kharkov National University of Radioelectronics. Her research interest includes the mathematical modeling and optimization in telecommunications. She is the coauthor of three books, author of more than 60 articles.

Sergey V. Garkusha received the $\mathrm{PhD}$ degree in telecommunication systems and networks in 2009. Currently, he is a docent department information activities in economic systems Poltava University of Economics and Trade and doctoral department of telecommunication systems Kharkov National University of Radioelectronics. His research interest includes the optimization and quality of service in wireless telecommunications. She is the coauthor of one books, author of more than 50 articles, and more than 3 inventions. 